\title{
Derechos de los robots ${ }^{1}$
}

\author{
Robots Law
}

Direitos dos robôs

Droits des robots

机器人的权利

Alfonso Celotto ${ }^{2}$ Università degli studi Roma Tre

Revista Derechos en Acción ISSN 2525-1678/ e-ISSN 2525-1686

Año 4/Nº 11 Otoño 2019 (21 marzo a 21 junio), 119-133

DOl: https://doi.org/10.24215/25251678e271

ORCID: https://orcid.org/0000-0003-0733-2334

Recibido: $18 / 05 / 2019$

Aprobado: 28/05/2019

Resumen: Durante décadas, hemos estado usando, teniendo, administrando refrigeradores, automóviles y teléfonos móviles. En suma, máquinas. Sin embargo, nadie ha pensado, hasta ahora, que tales máquinas podrían ser titulares de derechos. Las máquinas hasta ahora han sido "objetos" de derechos (propiedad, posesión, responsabilidad) y ciertamente no "sujetos". ¿Cómo es esto?

Por la sencilla razón de que solo recientemente estas máquinas han comenzado a tener formas propias de inteligencia artificial que empiezan a hacer dudar de que puedan asumir una cierta autonomía decisional. ¿Entonces estas máquinas pueden ser titulares de situaciones jurídicas propias?

\footnotetext{
1 Traducción del italiano al español realizada por Vannesa A. Pérez Rosales, quien es Juez Administrativo de Medellín (Colombia) y estudiante de doctorado en Derecho de la Universidad de Buenos Aires. La traductora agradece al Profesor Raúl Gustavo Ferreyra por sus invaluables aportes para la buena finalización de este trabajo.

2 Alfonso Celotto es Doctor en derecho constitucional y derecho público general de la Facultad de Jurisprudencia de la Universidad de Roma La Sapienza, profesor Ordinario de derecho constitucional de la Facultad de Jurisprudencia de la Universidad de Roma Tres y profesor invitado de la Universidad de Buenos Aires, de la Universidad de Varsovia y de la Universidad McGill de Montreal.
} 
Los robots de hace cien años no eran más que un invento literario de Karel Capek. Un invento fascinante para el desarrollo inmediato que los robots han tenido en la literatura de ciencia ficción de los siguientes años. Hasta el punto que un visionario como Asimov consiguiera establecer las leyes de la robótica. Era 1942. Pero hoy estamos realmente a punto de tener que escribir estas leyes. No solo porque están cambiando profundamente los derechos de las personas humanas, sino también porque están empezando a configurarse derechos propios de las máquinas.

Palabras claves: inteligencia artificial, robots, derechos.

Abstract: For decades, we have been using, managing refrigerators, automobiles and mobile phones. In short, machines. However, no one has thought, until now, that such machines could be rights holders. Machines so far have been "objects" of rights (ownership, possession, responsibility) and certainly not "subjects" of right. How is this?

For the simple reason that only recently these machines have begun to have their own forms of artificial intelligence that begin to make it doubtful that they can assume a certain decision-making autonomy. Can be these machines the holders of their own legal situations? The robots of a hundred years ago were nothing more than a literary invention of Karel Capek. A fascinating invention for the immediate development that robots have had in the science fiction literature of the following years. To the extent that a visionary like Asimov managed to establish the laws of robotics. It was 1942. But today we are really about to have to write these laws. Not only because the rights of human beings are profoundly changing, but also because the rights of machines are beginning to take shape.

Keywords: artificial intelligence, robots, rights.

Resumo: Durante décadas, temos estado usado, tendo, administando refrigeradores, carros e telefones celulares. Em suma, máquinas. No entanto, ninguém pensou, até agora, que essas máquinas poderiam ser titulares de direitos. As máquinas até agora têm sido "objetos" de direitos (propriedade, possessão, responsabilidade) e certamente não "sujeitos". Como é isso?

Pela simples razão de que só recentemente essas máquinas começaram a ter formas próprias de inteligência artificial que começam a fazer duvidar de que podem assumir certa autonomia de decisão. Então, essas 
máquinas podem ser titulares de situações legais próprias? Os robôs de cem anos atrás não eram mais que uma invenção literária de Karel Capek. Uma invenção fascinante para o desenvolvimento imediato que os robôs tiveram na literatura de ficção científica dos anos seguintes. A tal ponto que um visionário como Asimov conseguiu estabelecer as leis da robótica. Era 1942. Mas hoje estamos realmente a ponto de ter que escrever essas leis. Não só porque estão mudando profundamente os direitos das pessoas humanas, mas também porque estão começando a configurar-se direitos próprios das máquinas.

Palavras-chave: inteligência artificial, robôs, direitos.

Résumé: Pendant des décennies, nous avons utilisé, possédé et géré réfrigérateurs, voitures et téléphones portables. En bref, des machines. Cependant, personne n'a pensé jusqu'à présent que de telles machines pourraient bénéficier de droits. Les machines étaient jusqu'à présent des "objets" de droits (propriété, possession, responsabilité) et certainement pas des "sujets". Et pourquoi donc? Pour la simple raison que ce n'est que récemment que ces machines ont commencé à être dotées d'une forme propre d'intelligence artificielle; il pourrait en découler qu'elles puissent assumer une certaine autonomie décisionnelle. Donc, ces machines peuvent-elles être titulaires de situations juridiques propres? Les robots d'il y a cent ans n'étaient rien de plus qu'une invention littéraire de Karel Capek. Une invention fascinante vu le développement immédiat que les robots ont eu dans la littérature de science-fiction des années suivantes. Au point qu'un visionnaire comme Asimov a réussi à établir les lois de la robotique. C'était en 1942. Mais aujourd'hui, nous sommes vraiment sur le point de devoir écrire ces lois. Non seulement parce les droits des personnes humaines connaissent un profond changement, mais aussi parce que les machines commencent à bénéficier de droits propres.

Mot-clés: intelligence artificielle, robots, droits.

Sommario: Da decine di anni ciascuno di noi utilizza, possiede, gestisce frigoriferi, automobili, telefonini. In pratica, macchine. Eppure nessuno ha mai pensato, fino ad ora, che tali macchine potessero essere titolari di diritti.

Le macchine fino ad oggi sono state "oggetti" di diritti (proprietà, possesso, responsabilità) e non certo "soggetti". Come mai? 
Per la semplice ragione che soltanto di recente queste macchine hanno cominciato ad avere forme proprie di intelligenza artificiale che iniziano a far dubitare che possano assumere una certa autonomia decisionale.

Allora queste macchine possono essere titolari di situazioni giuridiche proprie?

I robot cent'anni fa altro non erano che una invenzione letteraria di Karel Capek. Una invenzione affascinante per l'immediato sviluppo che i robot hanno avuto nella letteratura fantascientifica degli anni successivi.

Al punto da far arrivare un visionario come Asimov a stabilire le leggi della robotica. Era il 1942. Ma oggi siamo davvero al punto di doverle scrivere queste leggi. Non solo perché si stanno modificando profondamente i diritti delle persone umane, ma anche perché stanno iniziando a configurarsi diritti propri delle macchine.

Parole chiave: intelligenza artificiale, robot, diritti.

摘要:几十年来, 我们一直在使用, 管理冰箱, 汽车和手机。简而言之, 机器。然而, 直到现在, 没有人想到这样的机器可能是权利的拥有 者。一百年前的机器人只不过是KarelCapek的文学发明。这是机器 人在接下来几年的科幻文学中所具有的直接发展的一项引人入胜 的发明。

至于像阿西莫夫这样的有远见的人设法建立了机器人法则。这是 1942年。但今天我们真的要写这些法律。这不仅是因为他们正在深 刻改变人类的权利, 而且因为他们开始配置自己的机器权利。

关键字: 人工智能, 机器人, 权利

\section{El reconocimiento}

¿Los robots deben tener sus propias leyes? El desarrollo de la tecnología está haciendo realidad la "predicción" hecha por Asimov en 1942: debemos establecer reglas para los robots, identificando también formas de capacidad jurídica, como si fueran auténticos sujetos de derechos ${ }^{3}$.

3 Todos deben recordar la formulación de Asimov en sus relatos sobre las tres leyes de la robótica, a las cuales luego se sumará la ley cero. 
Tradicionalmente, la regulación jurídica ha considerado a las máquinas solamente como "objeto" de derechos. En el sentido en que las leyes y reglamentos han regulado la propiedad, posesión, responsabilidad y traspaso de las máquinas usadas como herramientas.

Hoy, las máquinas se han vuelto cada vez más avanzadas. La disponibilidad de cantidades enormes de datos y de ordenadores cada vez más veloces ha llevado al desarrollo de máquinas dotadas de una nueva forma de inteligencia, no humana. Es decir, dotadas de algoritmos de "inteligencia artificial", que permiten el procesamiento de datos y un autoaprendizaje de alguna manera autónomos.

Así, los ordenamientos jurídicos se empiezan a plantear el problema de evaluar si las máquinas ya no pueden ser solo objetos, sino también sujetos de derechos. Quizás en la summa divisio de Aristóteles que distinguía los instrumentos a disposición del hombre para administrar su patrimonio en "instrumentos inanimados" e "instrumentos animados", pero ya los robots ya no pueden ser más clasificados simplemente entre los primeros.

\section{Porqué?}

Tomemos el caso de los vehículos autónomos.

En principio, los vehículos autónomos contradicen de manera flagrante todas las normas de tránsito vigentes, fundadas en el principio según el cual "Todo vehículo en movimiento o todo conjunto de vehículos en movimiento deberá tener un conductor." (art. 8 de la Convención de Viena sobre la Circulación Vial de 8 de noviembre de 1968). Pero el problema ciertamente no es este. En cuanto bastaría una redefinición de la categoría

"A robot may not injure a human being or, through inaction, allow a human being to come to harm.

A robot must obey any orders given to it by human beings, except where such orders would conflict with the First Law.

A robot must protect its own existence as long as such protection does not conflict with the First or Second Law" 
de vehículo. El problema es, sobretodo, la responsabilidad que los vehículos autónomos pueden generar. Tradicionalmente pensamos que un accidente de un vehículo autónomo puede ser regulado con las categorías clásicas de la responsabilidad. ¿Responsabilidad de quién? Ciertamente no del conductor que no existe. ¿Empero del propietario, del usuario, del fabricante o del programador?

Entonces quizá sea más simple pensar en un sistema de aseguramiento obligatorio. A cargo del propietario, del usuario, del fabricante o del programador. Como de hecho ha sido regulado ahora, por ejemplo en Alemania ${ }^{4}$. Pero pensamos que, en algún momento del desarrollo, estos vehículos serán cada vez más autónomos respecto al propietario (si todavía habrá uno), al usuario (podrán ser múltiples), al fabricante (¿responde también por los daños debidos a la circulación?) o al programador (probablemente no humano). Será cada vez más complejo determinar a cuál sujeto le incumbe la responsabilidad del resarcimiento o exigir de tal sujeto la reparación de los daños causados.

Por tanto, llegará a ser complicado utilizar las categorías clásicas del derecho y será más fácil pensar que la póliza del seguro se ponga a nombre... del mismo vehículo. Para entonces, el vehículo tendría su propia subjetividad jurídica. Por tanto, el vehículo autónomo podría, es más debería, tener también una cuenta corriente bancaria para pagar la póliza, recibir sus propias ganancias del servicio y hacer sus propias inversiones de capital $^{5}$. Una vez reconocidos el derecho de propiedad y la relativa administración autónoma del patrimonio a las máquinas,

4 En la primavera de 2017, la ley alemana sobre vehículos autónomos, que en todo caso aplica una regulación de tipo tradicional, pues exige que cuenten con un conductor con licencia que pueda tomar el control del vehículo, en caso de necesidad. Lo mismo ocurre en la regulación experimental de algunos Estados de E.E.U.U (California, Florida, Nevada). Elementos en: Palmerini, voz Robótica (parte giuridica), en: Enciclopedia di bioetica e scienza giuridica, X, Nápoles, 2016, 1104.

5 Cfr. Tegmark, Vita 3.0. Essere umani nell'era dell'intelligenza artificiale, Milán, 2018, 147 s. (N.T. Versión en español: Vida 3.0. Qué significa ser humano en la era de la inteligencia artificial, Taurus, 2018). 
le seguirían una serie de corolarios jurídicos (libertad personal, de circulación, derechos políticos legales a la pertenencia a la comunidad), y en últimas, el deber de pagar los impuestos, en una suerte de robotax $^{6}$, que configuraría una posición de imputabilidad autónoma, análoga del todo a la de una persona.

Aquí un robot tendría su subjetividad jurídica. Autónoma. Aunque con todas las dudas y las perplejidades del caso.

Antes de intentar afrontar el complejo problema, hace falta una precisión terminológica. Debo aclarar que hablaremos de "robots", también podríamos hablar de autómatas, humanoides o androides o más simplemente máquinas (pudiendo en este punto sostener que son en esencia todos sinónimos). Usamos "robots" recordando que la primera utilización del término data (solo) de 1920, en la obra del escritor checo Karel Capek, derivada de la palabra "robota" que significa "trabajo pesado", aunque podemos rastrear los primeros intentos de imaginar la figura de un robot a muchos siglos antes de Cristo, hasta llegar a Leonardo Da Vinci y a Frankestein.

De algún modo, no es fácil para el derecho definir qué es un "robot", en cuanto implica nociones de electrónica, mecánica e informática, no siempre simples ${ }^{7}$. Como quiera que, a nivel técnico para tener "robot" se requiere -básicamente- la concurrencia de dos elementos: movilidad y programabilidad, es decir, las máquinas deben ser capaces de moverse sobre la base de la programación autónoma. Tales características hacen autónomo al robot y reducen, hasta anular, los límites de la imputabilidad de los eventos a un posible operador humano.

\footnotetext{
6 Entre los primeros que ha hablado de esto Floridi, L. Robots, Jobs, Taxes, and Responsibilities. Philosophy \& Technology, 30(1), 2017, https://doi.org/10.1007/S13347-017-0257-3. En Italia, en este sentido se ha presentado en la XVII legislatura por el Excmo. Pastorelli, el PDL: Desgravaciones fiscales por el uso de sistemas de inteligencia artificial en la producción de bienes (Ley No. 4621 de 25 de septiembre de 2017).

7 Para un intento de definición, cfr. Palmerini, voce Robotica (parte giuridica), cit., 1100 s. Sobre la falta de una terminología precisa también: Bassini - Liguori - Pollicino, Sistemi di intelligenza artificiale, responsabilità, accountability. Verso nuovi paradigmi?, en Pizzetti, Intelligenza artificiale, protezione dei dati personali e regolazione, Turín, 2018, 335.
} 


\section{La focalización}

Dadas estas premisas, el primer problema jurídico a afrontar para comprender si los robots pueden ser sujetos de derechos es la titularidad jurídica para establecer "quien" puede ser titular de derechos.

Los derechos, como cualquier otra posición jurídica subjetiva, son atribuidos por el ordenamiento jurídico. En otras palabras, los derechos (subjetivos) son atribuidos por el derecho (objetivo). Con una precisión importante. El derecho objetivo puede atribuir derechos también a las personas no humanas, como ocurre con las personas jurídicas y, en algunas jurisdicciones, con los animales.

En los Estados contemporáneos, los titulares de derechos son, en general, los ciudadanos identificados por ordenamientos individuales, con precisiones y limitaciones para los extranjeros y también para algunas categorías de ciudadanos (como en el siglo XX la segregación racial en EEUU, el apartheid en Sudafrica, la cuestión aborigen en Australia). Partiendo de esta base, entonces las normas individuales adscriben el reconocimiento de derechos a las categorías de posibles titulares. Así en nuestro ordenamiento, son titulares de derechos también:

- los extranjeros y los apátridas (sent. No. 245/2011 de la Corte Constitucional);

- los ciudadanos de la Unión europea (Art. 20 Tratado de funcionamiento de la Unión Europea -TFUE);

- las personas no nacidas como el embrión y el concebido (art. 462 C.C.).

- las personas que ya no son capaces de entender o de querer, muy relevante para el problema del tratamiento de poner fin a la vida (piense en los casos de Englaro y Welby).

- las personas en estado de "sujeción especial" (como los detenidos).

- las personas jurídicas (salvo que por los derechos consustanciales a la corporalidad humana); 
- grupos y asociaciones (p.e. la protección del medio ambiente: cfr. Arts. 309 y 301 D.L. 152/2006).

- los animales (art. 13 TFUE).

Desde el punto de vista jurídico, nada obsta para que una forma de personalidad jurídica pueda ser reconocida también a sujetos o humanos, como pueden ser robots y máquinas. Después de todo, basta una línea de la ley para adscribir situaciones jurídicas subjetivas a las máquinas, pero cuando la cuestión es rica también en implicaciones filosóficas.

Vehículos autónomos, drones, sistemas de cirugía robótica, robots domésticos. Y también asistentes personales virtuales (que nos aconsejarán no solo el restaurante o el gimnasio, sino también la chica con quien salir, el trabajo a escoger y cómo votar en las elecciones), requerirán siempre de regulaciones más específicas y adecuadas.

Hasta ahora, de forma más tradicional y tranquilizadora, el derecho se ha limitado a regular los robots como "instrumentos", usando las categorías tradicionales de imputación de actos, omisiones y responsabilidades: quien quiera que sea fabricante, propietario, poseedor o usuario de un robot responde por sus actuaciones. Tomemos, por ejemplo, las Directivas UE 2001/95 y 2006/42 sobre la seguridad de los productos y las normativas sobre derechos y garantías del consumidor.

Pero ahora todo esto parece no ser suficiente.

Al día de hoy, la tentativa más avanzada de regular de forma orgánica el fenómeno "robot", está representado por la Resolución del Parlamento europeo de 16 de febrero de 2017 "con recomendaciones destinadas a la Comisión sobre normas de Derecho Civil sobre robótica” (2015/2103 (INL)), que pretende regular de modo uniforme para los Estados europeos, los aspectos civiles de la robótica, a partir de los aspectos de la responsabilidad.

Partiendo de los avances y la cada vez mayor autonomía de los robots (Considerandos $\mathrm{Z}, \mathrm{AA}, \mathrm{AB}$ ), la resolución considera que "las normas tradicionales no bastarán para generar 
responsabilidad jurídica por los daños ocasionados por el robot, ya que no permitirían determinar la parte que ha de hacerse cargo de la indemnización, ni exigir a dicha parte que repare el daño ocasionado" (Considerando AF).

He aquí la cuestión. La Resolución comprende que estamos avanzando hacia un modelo en el que será cada vez más difícil vincular la actividad de las máquinas con la de un solo responsable humano. Por tanto, hay que identificar una imputabilidad autónoma.

A nivel de regulación, la Resolución propone una serie de principios éticos, para asegurar que el desarrollo y la utilización de los robots suceda en condiciones de preservar la dignidad, la autonomía y la autodeterminación de los individuos y de garantizar la protección de la privacidad y que se preste atención "al posible desarrollo de un vínculo emocional entre seres humanos y robots, especialmente en el caso de grupos vulnerable (niños, ancianos, discapacitados), para mitigar los impactos emotivos y físicos". (punto 3).

Así, invita a afrontar la cuestión de los robots, antes que todo, desde el punto de vista de la responsabilidad, creando un sistema de registro de los robots, un aseguramiento obligatorio y un fondo de garantía para los daños causados por los robots no asegurados (puntos 53 y $\left.54^{8}\right)^{9}$.

Hasta aquí nos encontramos aún en la aplicación de las categorías tradicionales.

Pero, también pide al final (que se consideren soluciones como) "crear a largo plazo una personalidad jurídica específica para los robots, de forma que, como mínimo los robots autónomos más complejos puedan ser considerados personas electrónicas responsables de reparar los daños que puedan

8 N. de T. En la versión oficial de la Resolución en español, corresponde a los puntos 57 y 58.

9 Bassini - Liguori - Pollicino, Sistemi di intelligenza artificiale, responsabilità, accountability, cit., $345 \mathrm{~s}$. 
causar, y posiblemente aplicar la personalidad electrónica a aquellos supuestos en los que los robots tomen decisiones autónomas inteligentes o interactúen con terceros de forma independiente" (punto 59, literal f).

Entonces, ¿estamos preparados para crear una subjetividad electrónica?

Mucho más cauto a este respecto es el Dictamen del Comité Económico y Social de la Unión Europea (CESE, INT/086 de 31 de mayo de 2017), que se opone a atribuirle personalidad jurídica a los robots, en cuanto, "conllevaría a un riesgo moral inaceptable" 10 .

Volviendo a la Resolución, el Parlamento europeo también propone una Carta sobre Robótica, con una serie de principios éticos y deontológicos, dirigida ante todo a los investigadores, con el fin de garantizar una serie de principios fundamentales, que recuerdan mucho las leyes de Asimov: beneficencia, no perjuicio o maleficencia, autonomía, justicia ${ }^{11}$.

En suma, se trata, evidentemente, de una disciplina aún incierta, que busca sobre todo adaptar las categorías tradicionales al desarrollo de la robótica, pero con la plena conciencia de que alrededor de unas pocas décadas, los robots podrían alcanzar niveles de progreso hoy impensables (no olvidemos que hace 25 años internet no existía).

Por su parte, el Parlamento Italiano ha empezado a tomar conciencia del tema sobre todo desde el punto de vista

10 Punto 3.33. Sul dibattito cfr. Palmerini, voce Robotica (parte giuridica), cit., $1106 \mathrm{~s}$.

11 Específicamente se precisa:

- Beneficencia - los robots deben actuar en beneficio del hombre;

- Principio de no perjuicio o maleficencia - la doctrina de "primero, no hacer daño», en virtud del cual los robots no deberían perjudicar a las personas;

- Autonomía - la capacidad de tomar una decisión con conocimiento de causa e independiente sobre los términos de interacción con los robots;

- Justicia - la distribución justa de los beneficios asociados a la robótica y la asequibilidad de los robots utilizados en el ámbito de la asistencia sanitaria a domicilio y de los cuidados sanitarios en particular. 
cognoscitivo, como emerge, por ejemplo, del debate que la primavera de 2017 ha llevado la Cámara de los Diputados al exámen de algunas mociones sobre la robótica y la inteligencia $\operatorname{artificial}^{12}$.

\section{Los aspectos problemáticos}

De verdad nos encontramos frente a una gran revolución del orden social, antes incluso que normativa. Con fuertes incidencias sobre todos los modelos que conocemos.

De todos modos, hoy estamos solamente en una fase inicial y de transiciones: el desarrollo de la ciencia y de la tecnología no va a hacer más que ampliar tales problemáticas, extendiéndolas también a los aspectos de la intersección entre los derechos de las personas y los derechos de los autómatas.

A partir de los derechos de las personas. El problema del futuro de los derechos entretejido con la inteligencia artificial y el desarrollo de los robots ya había sido intuido por Bobbio, esperando "que la historia conduzca al Reino de los derechos del hombre en vez del Reino del Gran Hermano" "13. Es decir, los derechos humanos deben continuar siendo la garantía de la persona humana contra toda forma de poder y, desde luego, no pueden convertirse en instrumentos al servicio del poder $^{14}$. El

\footnotetext{
12 Cfr. moción Rosato y otros n. 1-01508, así como, en los textos reformulados respectivamente, las mociones Binetti y otros n. 1-01558, Cominardi y otros n. 1-01559, Rampelli y otros n. 1-01561, Ricciatti y otros n. 1-01562, Palese y otros n. 1-01571, Allasia y otros n. 1-01607, Catalano y otros n. 1-01608, Civati y otros n. 1-01619, Baldassarre y otros n. 101622 y Palmieri y Occhiuto n. 1-01623 sobre robótica e inteligencia artificial.

13 BoвBı, L'età dei diritti, Turín, 1990, 249. (N. de T. "El mundo de los hombres se dirige hacia la paz universal, como había previsto Kant, o hacia la guerra exterminadora, ipor esto ha sido acuñada, en oposición al pacifismo, uno de los ideales del siglo que creía en el progreso, la palabra exterminismo? ¿Hacia el reino de la libertad a través de un constante y cada vez más amplio movimiento de emancipación, de los individuos, de las clases y de los pueblos, o bien hacia el reino del Gran Hermano, descrito por Orwell?" En la edición en español: El tiempo de Ios derechos, Madrid: Ed. Sistema, 1991, pp. 176-177).

14 Cfr. también Cartabia, In tema di "nuovi" diritti, in Scritti in onore di F. Modugno, Nápoles, 2011, I, 643.
} 
riesgo es muy grande también porque todas las características de cada persona humana estarían concentradas en una serie de datos, a menudo también muy sensibles y detallados (hábitos, desplazamientos, tipologías de las personas contactadas, datos médicos y biométricos, etc), en posesión del banco de datos, sitio, proveedor: en la práctica, todo sobre cada uno de nosotros estará en nuestros datos. Datos en poder de una sociedad privada con fines comerciales. El riesgo no se protege solo con una tutela cada vez más rigurosa de la privacidad, como intenta hacer el nuevo reglamento de la UE sobre la protección de datos (679/2016), estableciendo reglas al derecho de rectificación, el derecho al olvido y el principio de cognoscibilidad y comprensibilidad del procesamiento automatizado (Cfr. Arts. 16, 17 y 22).

Hay que afrontar también el problema de la concentración de los datos, configurando -probablemente- mecanismos de separación y descentralización de los datos, para evitar que exista un solo poseedor de todos los datos de una persona y, en perspectiva, de todas las personas. Paradójicamente, avances y retrocesos históricos, el único modo para evitar el monopolio de los datos sería imponer mecanismos de descentralización, un poco como cuando para combatir el absolutismo se han impuesto las formas de división de los poderes.

Lo mismo sucederá con los datos y, por tanto, con los derechos de los robots. Podemos pensar que todos los robots estarían conectados en remoto con un solo cerebro conectado a la nube que conduciría y gestionaría todas las acciones? Parece peligroso, por varios aspectos, por tanto, también aquí se deberán adoptar protocolos de separación y descentralización.

Tenemos pues el problema de cuál sería la fuente idónea para regular tales derechos y situaciones jurídicas. ¿Quién podría hacerlo? Es dudoso pensar que pueden ser la Constitución y las leyes nacionales las que reglamenten los fenómenos que derivan del desarrollo tecnológico, como los robots ${ }^{15}$. Esta superación

15 Cfr. Bifulco, Intelligenza artificiale, internet e ordine spontaneo, en Pizzetti, Intelligenza artificiale, protezione dei dati personali e regolazione, Turín, 2018, p. 381-ss. 
de la soberanía regulatoria nacional sobre los fenómenos tecnológicos parece moverse en dos direcciones: de un lado, hacia la exigencia de parámetros constitucionales y regulatorios globales, de otro lado, con espacios cada vez mayores por las necesidades de autonomía de los propios sistemas, por lo que al legislador le será cada vez más difícil perseguir las continuas innovaciones tecnológicas. Aquí se abren los espacios hacia la teoría del orden espontáneo de Hayek, pero también con auto regulación de los mismos "gigantes" de la red, en cuanto cada vez más a menudo serán los productores individuales quienes pongan las reglas. Tendremos cada vez más normativas no "heterónomas"; en el sentido de que serán formuladas por el mismo fabricante o gestor de la nueva tecnología, como ya ocurre con las "reglas éticas" planteadas por cada una de las grandes compañías particulares ${ }^{16}$.

Después de todo, los ordenamientos estatales son normalmente misoneístas y tardígrados. Aún más en sectores de rapidísima obsolescencia como la tecnología. Casos emblemáticos pueden ser el cigarrillo electrónico, producto nuevo que ha luchado para ser referido a las categorías tradicionales, como productos para fumar o dispositivos médicos ${ }^{17}$.

En el sentido que, en nombre del principio básico tradicional "ubi societas, ibi ius", el derecho del Estado es siempre más lento y se adapta con dificultad a las novedades. Incluso más cuando son novedades que crecen y se desarrollan siguiendo el principio de singularidad tecnológica, es decir, con una aceleración más allá de cualquier comprensible capacidad de anticipación humana.

\footnotetext{
16 Piénsese no solo en los códigos éticos ya adoptados por empresas particulares, como por ejemplo IBM y Volvo Google, sino también por los acuerdos colectivos para prohibir de manera absoluta robots asesinos.

17 , en el pasado, la limitación de los automóviles mediante el Locomotives Act inglés del $1^{\circ}$ de agosto de 1861, con límites de velocidad rigurosísimos. 0 el de la televisión gratuita en Italia obra del D.P.R. 29 marzo 1973, n. 156, que reforzó el monopolio de la RAI, imponiendo una autorización estatal para todas las formas de telecomunicaciones.
} 
Si realmente existe este desarrollo, en el que las formas de la inteligencia artificial y, por tanto, las capacidades de los robots alcanzarán y superarán las humanas, debemos preguntarnos si para los robos serían aplicables las reglas pensadas para seres humanos, o si emergerá la exigencia de que los mismos robots definan las reglas aplicables a los robots. Respetar un principio de "auto-declaración" como forma de posible pluralismo.

¿Llegaremos a un derecho de los robots posicionado al nivel más o menos global de los mismos robots?

De cualquier modo, la regulación de los derechos de los robots implica toda una serie de preguntas, de las cuales por el momento aún no alcanzamos a ver las posibles soluciones. También si, tratándose de terrenos tan innovadores, se llegase a pensar que probablemente emergerán también cuestiones ulteriores, que hoy no alcanzamos ni siquiera a imaginar. Básicamente, se trata de una revolución histórica, un poco como cuando aparece la exigencia de regular el derecho de los mares y se pensó que era imposible hacerlo con el simple nomos de la tierra $^{18}$, como hoy no se puede pensar en regular la red con los instrumentos legislativos tradicionales ${ }^{19}$.

De cualquier modo, parece necesaria una aproximación global e interdisciplinaria. Porque ciertamente no basta la visión limitada, para regular fenómenos del todo innovadores, de las estrechas categorías del derecho.

\footnotetext{
18 Schmiтt, II nomos della terra nel diritto internazionale dello "jus publicum europaeum" (1950), ed. it., Milán, 1991. (N. del T. Versión en español: El nomos de la tierra en el Derecho de Gentes del "lus publicum europaeum", Granada: Ed. Comares, 2002).

19 Marongiu, Organizzazione e diritto di internet, Milán, 2013; y últimamente, CARotTı, II sistema di governo di internet, Milán, 2016.
} 